\title{
Atomistic modeling and experimental studies of radiation damage in monazite-type $\mathrm{LaPO}_{4}$ ceramics
}

\author{
Yaqi Ji ${ }^{\mathrm{a}, \mathrm{b}}$, Piotr M. Kowalski ${ }^{\mathrm{a}, \mathrm{b}, *}$, Stefan Neumeier ${ }^{\mathrm{a}, \mathrm{b}}$, Guido Deissmannn, ${ }^{\mathrm{a}, \mathrm{b}}$, Pawan K. Kulriya ${ }^{\mathrm{c}, \mathrm{d}}$, Julian D. Gale \\ ${ }^{a}$ Institute of Energy and Climate Research (IEK-6), Forschungszentrum Jülich, Wilhelm-Johnen-Straße, 52425 Jülich, Germany \\ ${ }^{b}$ JARA High-Performance Computing, Schinkelstraße 2, 52062 Aachen, Germany \\ ${ }^{c}$ Inter-University Accelerator Centre (IUAC), Aruna Asaf Ali Road, New Delhi 110067, India \\ ${ }^{d}$ Department of Mechanical, Aerospace $\mathcal{E}$ Nuclear Engineering, Rensselaer Polytechnic Institute, Troy, NY 12180, United States \\ ${ }^{e}$ Curtin Institute of Computation, Department of Chemistry, Curtin University, PO Box U1987, Perth, WA 6845, Australia
}

\begin{abstract}
We simulated the threshold displacement energies $\left(E_{d}\right)$, the related displacement and defect formation probabilities, and the energy barriers in $\mathrm{LaPO}_{4}$ monazite-type ceramics. The obtained $E_{d}$ values for La, $\mathrm{P}, \mathrm{O}$ primary knock-on atoms (PKA) are $56 \mathrm{eV}, 75 \mathrm{eV}$ and $8 \mathrm{eV}$, respectively. We found that these energies can be correlated with the energy barriers that separate the defect from the initial states. The $E_{d}$ values are about twice the values of energy barriers, which is explained through an efficient dissipation of the PKA kinetic energy in the considered system. The computed $E_{d}$ were used in simulations of the extent of radiation damage in $\mathrm{La}_{0.2} \mathrm{Gd}_{0.8} \mathrm{PO}_{4}$ solid solution, investigated experimentally. We found that this lanthanide phosphate fully amorphises in the ion beam experiments for fluences higher than $\sim 10^{13}$ ions $/ \mathrm{cm}^{2}$.
\end{abstract}

Keywords:

Radiation damage; Ceramic materials; Molecular dynamics; Threshold displacement energy; Energy barrier; Irradiation experiments; Nuclear waste management;

\section{Introduction}

Monazites are rare-earth phosphate minerals $\left(\operatorname{Ln} \mathrm{PO}_{4}\right)$ that occur in nature often containing significant amounts of radioactive elements, such as Th or $\mathrm{U}$, without indication of significant radiation damage imposed on their crystalline structures [1]. Being chemically durable monazite-type ceramics are considered as candidate materials for nuclear waste disposal form suitable for long term immobilization of actinides, in particular plutonium $[2,3,4]$. Therefore, various relevant properties of these materials have been extensively investigated. These include the structural, the thermochemical and the thermodynamic parameters (e.g. [5, 6, 7, 8, 9, 10, $11,12,13,14,15])$ as well as the dissolution [16], the elastic $[17,10]$ and the radiation damage properties $[18,19]$.

Threshold displacement energy $\left(E_{d}\right)$ is a minimum kinetic energy required to displace an atom from its lattice site. It is a fundamental parameter used to define

${ }^{*}$ Corresponding author: Piotr M. Kowalski Tel.: +49 246161 9356, E-mail: p.kowalski@fz-juelich.de the radiation tolerance of materials and to estimate the extend of radiation damage during a radiation process, using for instance software such as Stopping and Range of Ions in Matter (SRIM) [20, 21, 22]. Because of the short, ps time-scale of the radiation cascade processes, atomistic modeling is a good tool to obtain the values of $E_{d}$, which otherwise is challenging to experimental methods. Such simulations have been performed recently for many materials, including $\mathrm{TiO}_{2}$ rutile [20], $\mathrm{ZrO}_{2}$ [23], $\mathrm{BaTiO}_{3}$ [24], $\mathrm{SrTiO}_{3}$ [25], or graphene and carbon nanotubes [26], to name but a few.

To displace an atom permanently, there are energy barriers separating the initial state and the final defect state in materials. Knowing the final state, these barriers can be calculated using, for instance, the nudged elastic band (NEB) method, but can be also traced during simulations of the $E_{d}$ values. In previous study of radiation damage in diamond, Wu \& Fahy [27] found that the damage threshold energy is almost twice the sum of bond-breaking and crystal strain energy due to the efficient dissipation of the kinetic energy of primary knock-on atom (PKA) to the crystalline lattice vibra- 
Table 1: The Buckingham potential parameters used in the simulations. [7]

\begin{tabular}{lccc}
\hline & $\mathrm{A}(\mathrm{eV})$ & $\mathrm{B}(\AA)$ & $\mathrm{C}\left(\AA^{6} \cdot \mathrm{eV}\right)$ \\
\hline La-O & 17927 & 0.25934 & 0.0000 \\
Gd-O & 13271 & 0.26 & 0.0000 \\
$\mathrm{P}-\mathrm{O}$ & 877.3 & 0.3594 & 0.0000 \\
$\mathrm{O}-\mathrm{O}$ & 22764.3 & 0.1490 & 27.879 \\
\hline
\end{tabular}

tions. However, the question if this is intrinsically related to diamond or a general property of materials remains open.

In this contribution we derived the $E_{d}$ values and the related displacement and defect formation probabilities for $\mathrm{LaPO}_{4}$ monazite-type ceramics and compare the results with the recent studies of $\mathrm{TiO}_{2}$ rutile [20]. The obtained $E_{d}$ values were subsequently used in simulations of extend of radiation damage in $\mathrm{La}_{0.2} \mathrm{Gd}_{0.8} \mathrm{PO}_{4}$ monazite-type solid solution in order to help in setting up the proper conditions of the irradiation experiments. We also report our first results on the ion beam irradiation of this material.

\section{Computational and experimental details}

The simulations of $E_{d}$ values were performed with 104 the LAMMPS code using, in addition to the standard 105 Coulomb interaction term, the Buckingham-type inter- 106 action potentials,

$$
\Phi_{12}=A \exp (-B r)-C / r^{6}
$$

which $A, B$ and $C$ parameters for $L n-O$ interactions have 112 been fitted so the classical simulation reproduce the $a b{ }_{113}$ initio data of Blanca-Romero et al. [7], and the parame- 114 ters for P-O and O-O interaction are the ones of Gale \& ${ }_{115}$ Henson [28] and Girard et al. [29]. All the parameters 116 are given in Table 1.

We simulated the PKA $E_{d}$ values and the displace- ${ }_{118}$ ment and defect formation probabilities in the PKA en- 119 ergy range of $50-150 \mathrm{eV}$ for $\mathrm{La}$, of $75-250 \mathrm{eV}$ for $\mathrm{P}$ and ${ }_{120}$ of 8-50 eV for $\mathrm{O}$. The simulations were performed with ${ }_{121}$ the supercells containing 1536 atoms and for each PKA 122 energy we performed 100 independent simulations with 123 the PKA initial velocity directions distributed randomly 124 and symmetrically on a surface of a sphere using the 125 Thompson model [22]. In our simulations both methods 126 yielded very similar results. All the simulations were $5{ }_{127}$ ps long which was enough for the diminishing of the effect of the initial cascade and subsequent equilibration of the system. In order to estimate the displacement probability and the defect formation probabilities we used an algorithm to analyze displacements and defects according to the initial and final positions of atoms in the lattice. These simulations were performed with $T=300 \mathrm{~K}$, controlled by a thermal layer.

The subsequent calculations of energy barriers and the defect states were performed using NEB and metadynamics methods. The NEB calculations were performed with the relevant package implemented in the LAMMPS code [30] and the metadynamics simulations were performed with the PLUMED plug-in [31].

The penetration depth of the ions, the resulting displacements of target atoms and the distribution of vacancies in the experimentally studied $\mathrm{La}_{0.2} \mathrm{Gd}_{0.8} \mathrm{PO}_{4}$ system were calculated with the SRIM/TRIM software package, using the SRIM-2013 code (www.srim.org). SRIM/TRIM (Stopping and Range of Ions in Matter/Transport of Ions in Matter) comprises a set of programs that can simulate the interactions of ions with energies up to $2 \mathrm{GeV} / \mathrm{amu}$ with matter, based on a full quantum mechanical treatment of the collisions of incident particles with atoms present in a target material $[32,33]$. The code is based on a Monte Carlo (MC) simulation method and the binary collision approximation (BCA) [34, 35]. Simulation results comprise, for example, the 3D-distribution of ions and the concentration of vacancies in the target material as well as the energy partitioning between nuclear and electronic energy losses, with all target atom cascades in the target material followed in detail. SRIM/TRIM generally assumes that the target is isotropic and amorphous.

For the irradiation experiments a highly densified $\left(\rho_{\text {sint }}=97 \%\right.$ of theoretical density (TD) $) \mathrm{La}_{0.2} \mathrm{Gd}_{0.8} \mathrm{PO}_{4}$ pellet of $10 \mathrm{~mm}$ diameter and $1 \mathrm{~mm}$ thickness has been prepared according to Neumeier et al. [36] and Arinicheva et al. [37]. The purity of the monazite sample material was confirmed by the XRD measurements (Bruker D8-Advance X-ray diffractometer (XRD)). The pellet was irradiated at room temperature with $100 \mathrm{MeV}$ ${ }^{197} \mathrm{Au}^{9+}$ ions delivered by the 15 UD Pelletron accelerator at the Inter-University Accelerator Centre (IUAC) Delhi, India at the ion fluence ranging from $10^{12}$ ions $/ \mathrm{cm}^{2}$ to $2 \cdot 10^{14}$ ions $/ \mathrm{cm}^{2}$. The ion flux was kept below $2.8 \cdot 10^{10}$ ions $\mathrm{cm}^{-2} \mathrm{~s}^{-1}$ in order to avoid ion beam induced heating of the target materials. A Bruker D8XRD was used for in-situ investigations of the irradiation induced structural modifications $[38,39]$. The in-situ experiments were performed on the same pellet by successive irradiation and the immediate subsequent 


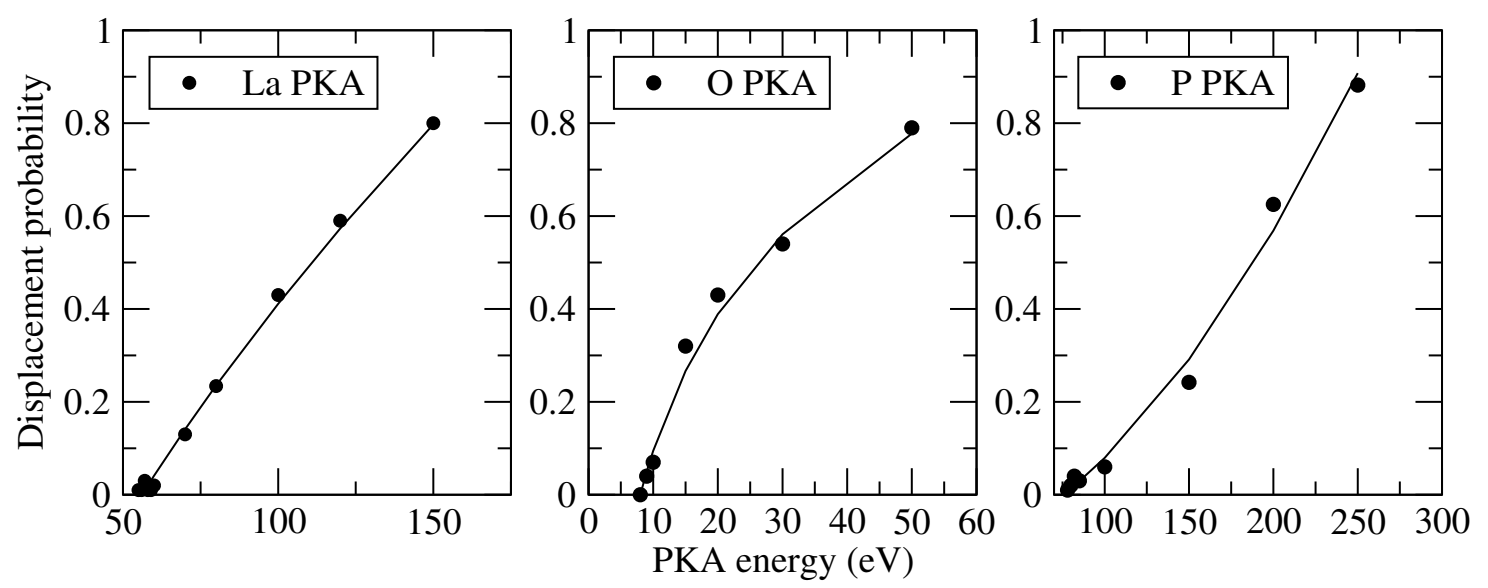

Figure 1: The atom displacement probabilities of $\mathrm{La}, \mathrm{O}, \mathrm{P}$ PKA in $\mathrm{LaPO}_{4}$ simulated at $T=300 \mathrm{~K}$.

XRD measurement without changing experimental parameters in order to compare the intensity of the diffraction reflections of the sample exposed to the different ion fluences. All XRD patterns were recorded under vacuum $\left(5 \cdot 10^{-6} \mathrm{mbar}\right)$ in the $2 \theta$ range of $10-90^{\circ}$ with increments of $0.02^{\circ}$ at a scan speed of $0.5^{\circ} \mathrm{min}^{-1}$.

\section{Results and discussion}

\subsection{Threshold displacement energy of $\mathrm{LaPO}_{4}$}

The displacement probabilities as a function of PKA energy for La, $\mathrm{P}$ and $\mathrm{O}$ atoms are shown in Figure 1. In the figure each point is the average value obtained by sampling the 100 PKA directions. The threshold displacement energy can be obtained from the relationship between the initial energy and the displacement probabilities by fitting the equation $[20,22]$ :

$$
D P(E)=\left[E^{\alpha}-E_{d}^{\alpha}\right] / \beta, E>E_{d},
$$

where $\alpha, \beta$ and $E_{d}$ are the fitting parameters and $E$ is the PKA energy. The $E_{d}$ value fitted for La is $56 \mathrm{eV}$, for $\mathrm{P}$ is $75 \mathrm{eV}$ and for $\mathrm{O}$ is $8 \mathrm{eV}$. These values indicate that it is easiest to form an $\mathrm{O}$ defect and hardest to form a $\mathrm{P}$ defect in the $\mathrm{LaPO}_{4}$ lattice. This is because in $\mathrm{LaPO}_{4}$, one $\mathrm{P}$ atom is bonded with four $\mathrm{O}$ atoms and one $\mathrm{PO}_{4}$ is interacting with one $\mathrm{La}$ atom, which results in the different bounding strengths and resulting $E_{d}$ values. Interestingly, the $E_{d}$ value for La is similarly large as the one obtained for $\mathrm{Ti}$ cation in $\mathrm{TiO}_{2}$ rutile $(69 \mathrm{eV},[20]) . \quad{ }_{169}$ Also, the difference between the displacement probabil- 170 ity and the defect formation probability obtained in our 171 studies, and shown in Figure 2, is very similar to the 172 one obtained for rutile. Namely, the defect formation 173

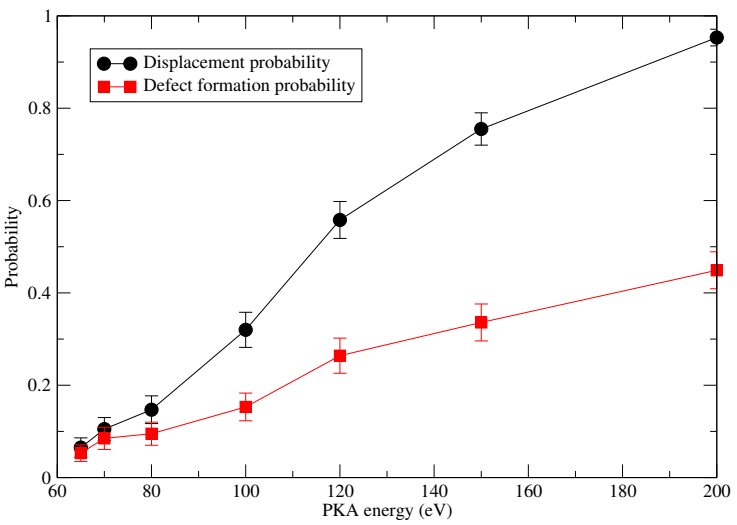

Figure 2: The displacement probabilities and the defect formation probabilities for La cations in $\mathrm{LaPO}_{4}$ as a function of PKA energy, simulated at $T=300 \mathrm{~K}$.

probability is significantly smaller and our results indicate that at a temperature of $300 \mathrm{~K}$ about half of the La displacements recombine to a regular La crystalline position. In the case of rutile, Robinson et al. [20] attributed the radiation damage resistance of this material to its efficient defect recombining ability. Our similar results indicate thus a possibility of a common origin of radiation damage resistance in the case of rutile and monazite. The $E_{d}$ value obtained for the Gd cation with the same method and used in the SRIM simulations (see section 3.3 ) is $51 \mathrm{eV}$.

\subsection{Energy barriers in displacement of $\mathrm{LaPO}_{4}$}

Formation of permanent defects is related to the energy barrier $\left(E_{b}\right)$ that has to be crossed by a PKA atom. Therefore, we checked how the energy barrier, defined here as the minimum potential energy increase (maximum) during the cascade, correlates with the initial 


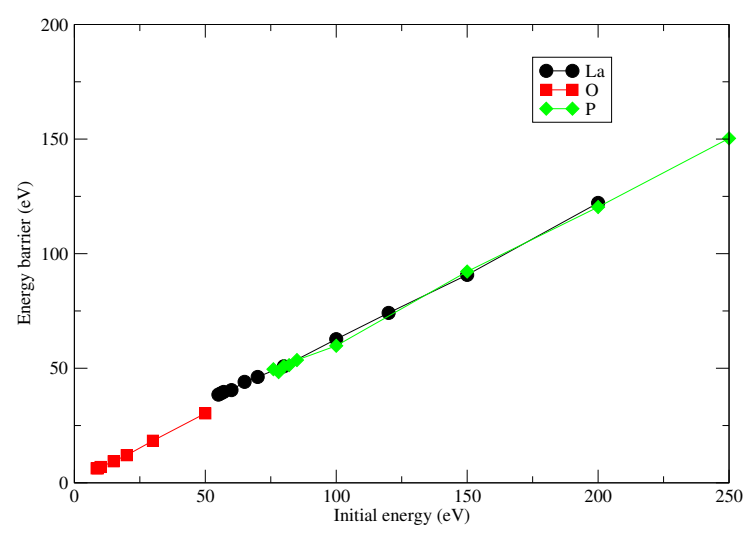

Figure 3: The relationship between the PKA energy and the energy barrier in $\mathrm{LaPO}_{4}$. Results for all three species are plotted.

PKA energy and the $E_{d}$ value. The relationships for the three cations considered are presented in Figure 3. We found that the energy barriers are substantially smaller, 209 by about a half, than the applied initial PKA energies 210 and there is no defect created, if the PKA energy is just 211 comparable to the energy barrier. As shown in Figure 3, 212 there is a linear relationship between the energy barrier 213 and the PKA energy, $E_{b} \sim 0.58 E$, and the relationships 214 are very similar for all the three considered species. 215 This result has been verified with the subsequent cal- 216 culations of barriers performed by a combination of the 217 NEB and metadynamics methods. Interestingly, very 218 similar results have been reported for diamond by $\mathrm{Wu} 219$ \& Fahy [27], who also found that the PKA energy must 220 be about twice the energy barrier to overcome the bar- 221 rier. They attempted an explanation of this phenomenon 222 by invoking similarity of the initial PKA velocity to the 223 speed of sound, which allows for efficient transfer of the 224 PKA kinetic energy to the energy of lattice vibrations. 225 Therefore, we performed a detailed analysis of the dis- 226 sipation of the initial PKA kinetic energy in the system 227 studied.

The evolution of kinetic and potential energies in the ${ }^{229}$ two cases: (1) without defect and (2) with defect for- ${ }^{230}$ mation is illustrated in Figure 4. In the case without ${ }^{231}$ the defect, the PKA energy is equally distributed to the ${ }^{232}$ kinetic energy of other atoms and the potential energy ${ }^{233}$ of entire system. The case with the defect creation is a ${ }^{234}$ little bit different. Initially, the PKA kinetic energy is also equally distributed between the kinetic and potential energies of the system but after crossing the barrier and equilibration, the gain in the kinetic energy of the 236 system is smaller than the gain in the potential energy. 237 In the considered case, the difference is about $12 \mathrm{eV} . \quad 238$ This value is independent of the initial PKA kinetic en- 239

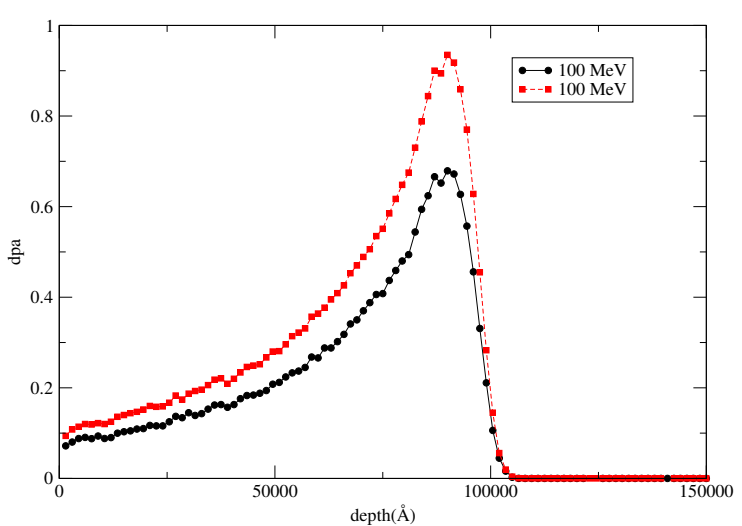

Figure 5: The relationship between defect extent (in dpa) and the ion range computed by SRIM assuming bombardment of $\mathrm{LaPO}_{4}$ with 100 $\mathrm{MeV} \mathrm{Au}$ ions and a fluence of $10^{14}$ ions $/ \mathrm{cm}^{2}$. The results for $E_{d}$ values (black circles) and energy barriers $\left(E_{b}=0.58 E_{d}\right.$, red squares) the computed here are presented.

ergy and is equal to the defect formation energy, which we verified through subsequent relaxation of the final state.

Having this result and following the studies of $\mathrm{Wu}$ \& Fahy [27], we compared the PKA velocities to the speed of sound in $\mathrm{LaPO}_{4}$ monazite. The sound velocity in $\mathrm{LaPO}_{4}$ can be calculated from the knowledge of bulk modulus, shear modulus and material density. For $\mathrm{LaPO}_{4}$ monazite, it is about $3664 \mathrm{~m} / \mathrm{s}$ [17], which means that the sound waves can travel through the supercell in just $\sim 0.5 \mathrm{ps}$ and the corresponding energy is $\sim 10 \mathrm{eV}$. Thus, a La PKA atom with the energy of the threshold displacement energy of $56 \mathrm{eV}$ has a velocity of 8864 $\mathrm{m} / \mathrm{s}$, which is comparable to the above-provided speed of sound. This explains why a significant part $(\sim 50 \%)$ of the PKA energy is efficiently transferred into the system and dissipated through the lattice vibrations.

Finding a relationship between the PKA energy, the $E_{d}$ values and the energy barriers can be very useful for determination of the $E_{d}$ values. This is because computation of barriers is computationally less demanding and provides an independent way to estimate the $E_{d}$ values. For instance, the defect states could be identified with methods such as metadynamics, and the barrier between the initial ground state and the defect state could, for instance, be computed with NEB method.

\subsection{Simulation of radiation damage extent with SRIM}

The obtained $E_{d}$ values have been used in subsequent simulations of the extent of radiation damage under conditions reflecting the planned irradiation experiments. We also made computations taking energy barriers as 

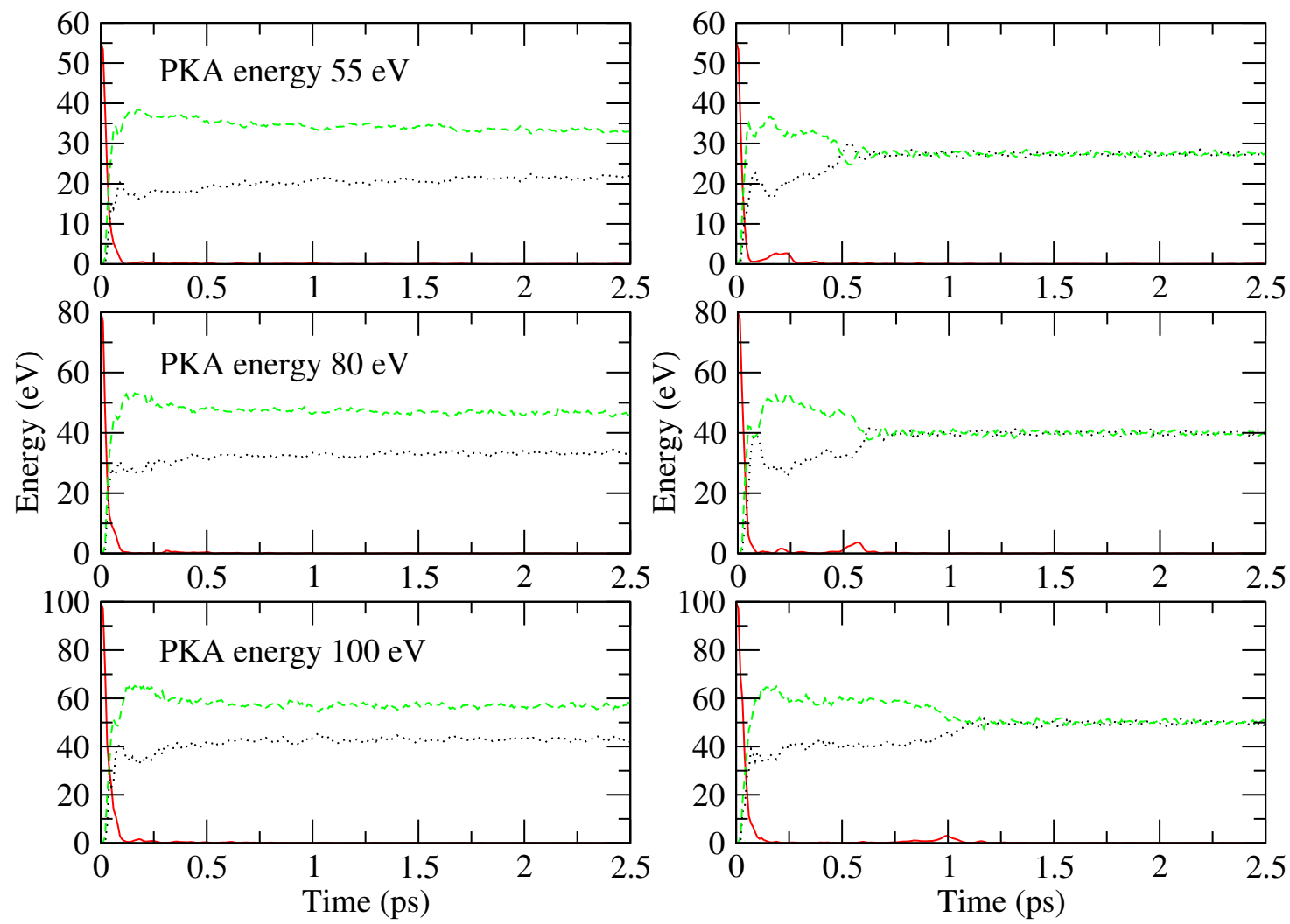

Figure 4: The kinetic energy of the PKA (solid red), the kinetic (dotted black) and potential (dashed green) energy of all the atoms except PKA atom, obtained with different initial PKA energies indicated in the upper left corners. The left panels are results obtained for cases when a defect was created and the right side panels represent the results obtained without defect creation.

$E_{d}$ values, thus reducing the $E_{d}$ values to $0.58 E_{d}$. Figure 5 shows the results of such simulations. These indicate that the expected radiation dose expressed in displacements per atom (dpa) is higher than the critical amorphization dose reported for monazites $(\sim 0.35 \mathrm{dpa}$, $[18,2])$. Thus it was ascertained that the maximum fluence selected in the irradiation experiments would be sufficiently high to allow for the amorphization of the monazite samples. The damage peaks at the depth of $9 \mu \mathrm{m}$ and thus should be easily detectable by XRD techniques. Also, the results of simulations with the two sets of $E_{d}$ values are consistent regarding the penetration range and differ only in prediction of the damage amount, when smaller $E_{d}$ values are used.

\subsection{XRD measurement}

The XRD measurements of the $\mathrm{La}_{0.2} \mathrm{Gd}_{0.8} \mathrm{PO}_{4}$ solid solution sample irradiated with the $100 \mathrm{MeV}$ Au ions at ${ }_{261}$ fluences ranging from $10^{12} \mathrm{ions} / \mathrm{cm}^{2}$ to $10^{14}$ ions $/ \mathrm{cm}^{2}{ }_{262}$ agree with the SRIM calculations (Figure 6). Compared 263 with the XRD pattern of unirradiated material, the XRD ${ }_{264}$ reflections of irradiated samples become broader and 265

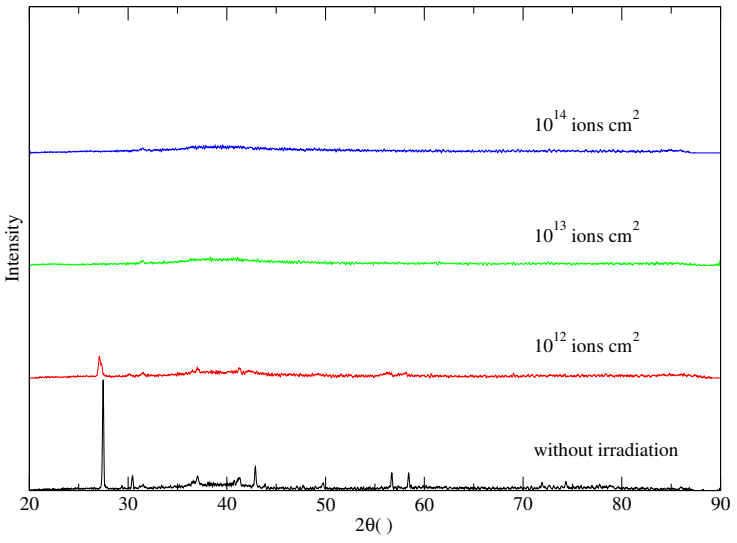

Figure 6: The XRD of $\mathrm{La}_{0.2} \mathrm{Gd}_{0.8} \mathrm{PO}_{4}$ solid solutions irradiated with $100 \mathrm{MeV} \mathrm{Au}$ ions at different fluences.

vanish gradually at higher fluences $\left(10^{13} \mathrm{ions} / \mathrm{cm}^{2}\right)$, indicating complete amorphization. However, amorphization was achieved already at a lower fluence than predicted from the SRIM results. This effect was already observed in irradiation experiments with pyrochlore- 
type materials using swift heavy ions and is due to 314 the thermal spike induced by electronic stopping effects 315 [40].

\section{Conclusion}

Using atomistic modeling techniques we simulated the radiation damage resistance of the $\mathrm{LaPO}_{4}$ monazitetype ceramics. We derived the $E_{d}$ values for all three species constituting the investigated material. These ${ }_{32}$ values are largest for $\mathrm{P}(75 \mathrm{eV})$, significant for $\mathrm{La}(56$ $\mathrm{eV})$ and relatively small for $\mathrm{O}(8 \mathrm{eV})$. Interestingly, the ${ }^{323}$ value obtained for La is similarly large as the one de- ${ }_{325}^{324}$ rived for the Ti cation in $\mathrm{TiO}_{2}$. Also, the obtained dif- ${ }_{326}$ ference between the displacement and defect formation ${ }^{327}$ probabilities derived for $\mathrm{La}$ in monazite is very similar ${ }^{328}$ to the results obtained for $\mathrm{Ti}$ in rutile $\mathrm{TiO}_{2}$, which points ${ }_{330}^{329}$ towards a similar origin of the radiation damage resis- ${ }_{33}$ tance of both materials. We found a linear relationship ${ }^{332}$ between the energy barriers separating the initial from ${ }_{334}^{333}$ the defect state and the PKA initial energy values, which ${ }_{335}^{334}$ indicates that the barrier could be crossed only if the 336 PKA energy is about twice the barrier energy. This we ${ }^{337}$ explain by efficient dissipation of the PKA kinetic en- ${ }_{339}^{338}$ ergy between the potential energy and the kinetic energy ${ }_{340}$ of vibration of the crystalline. The obtained $E_{d}$ values ${ }^{341}$ have been applied to simulations of radiation damage ${ }^{342}$ extent under various experimental conditions, helping ${ }_{344}^{343}$ selecting proper setup parameters for the irradiation ex- ${ }_{345}$ periments. The irradiation experiments and subsequent ${ }^{346}$ XRD measurements of the irradiated samples indicate ${ }^{347}$ full amorphization of the samples for fluences higher ${ }^{348}$ than $10^{13}$ ions $/ \mathrm{cm}^{2}$. The subsequent experimental and 350 modeling studies are ongoing in order to improve our 351 understanding of the radiation-induced amorphization process in monazites.

\section{Acknowledgement}

YJ is thankful to China Scholarship Council (CSC) ${ }_{35}^{35}$ for providing financial supports for her PhD study in 360 Germany at Forschungszentrum Jülich/RWTH Aachen. ${ }^{361}$ SN and PKK acknowledge financial support from the ${ }^{362}$ DSTDAAD under a joint research project grant No. ${ }_{364}$ INT/FRG/DAAD/P-02/2016. JDG thanks the Aus- 365 tralian Research Council for funding through the Dis- ${ }^{366}$ covery Programme, as well as the Pawsey Supercom- ${ }^{367}$ puting Centre and National Computational Infrastruc- ${ }_{369}$ ture for provision of computing resources. Funded 370 by the Excellence Initiative of the German federal ${ }^{371}$ and state governments and the Jülich Aachen Re- ${ }_{373}^{372}$ search Alliance - High-Performance Computing. We ${ }_{374}$ thank the JARA-HPC awarding body for time on the RWTH and Forschungszentrum Jülich computing resources awarded through JARA-HPC Partition. We gratefully acknowledge the funding from the German Federal Ministry of Education and Research (BMBF, grant 02NUK021A).

\section{References}

[1] C. M. Gramaccioli, T. V. Segalstad, Am. Mineral. 1978, 63, 757.

[2] R. Ewing, L. Wang, in Phosphates: Geochemical, Geobiological, and Materials Importance, M. Kohn, and J. Rakovan, and J. Hughes, ed., Mineral Soc. Amer., 2002, Reviews in Mineralogy \& Geochemistry, vol. 48, (pp. 673-699).

[3] G. Deissmann, S. Neumeier, G. Modolo, D. Bosbach, Mineralogical Magazine 2012, 76, 2911.

[4] H. Schlenz, J. Heuser, A. Neumann, S. Schmitz, D. Bosbach, Z. Kristallogr. 2013, 228, 113.

[5] N. Clavier, R. Podor, N. Dacheux, J. Eur. Ceram. Soc. 2011, 31, 941.

[6] S. Ushakov, K. Helean, A. Navrotsky, J. Mater. Res. 2001, 16, 2623.

[7] A. Blanca-Romero, P. M. Kowalski, G. Beridze, H. Schlenz, D. Bosbach, J. Comput. Chem. 2014, 35, 1339.

[8] P. M. Kowalski, G. Beridze, V. L. Vinograd, D. Bosbach, J. Nucl. Mater. 2015, 464, 147.

[9] G. Beridze, A. Birnie, S. Koniski, Y. Ji, P. M. Kowalski, Progress in Nuclear Energy 2016, 92, 142 .

[10] P. M. Kowalski, Y. Li, J. Eur. Ceram. Soc. 2016, 36, 2093

[11] A. Thust, Y. Arinicheva, E. Haussühl, J. Ruiz-Fuertes, L. Bayarjargal, S. C. Vogel, S. Neumeier, B. Winkler, J. Am. Ceram. Soc. 2015, 98, 4016.

[12] Y. Li, P. M. Kowalski, A. Blanca-Romero, V. Vinograd, D. Bosbach, J. Solid State Chem. 2014, 220, 137 .

[13] P. M. Kowalski, G. Beridze, Y. Li, Y. Ji, C. Friedrich, E. Sasioglu, S. Blügel, Ceram. Trans. 2016, 258, 207.

[14] J. Heuser, A. Bukaemskiy, S. Neumeier, A. Neumann, D. Bosbach, Progress in Nuclear Energy 2014, 72, 149.

[15] S. Neumeier, P. Kegler, Y. Arinicheva, A. Shelyug, P. M. Kowalski, A. Navrotsky, D. Bosbach, Submitted 2016, XX, XX.

[16] F. Brandt, S. Neumeier, T. Schuppik, Y. Arinicheva, A. Bukaemskiy, G. Modolo, D. Bosbach, Prog. Nucl. Energ. 2014, 72, 140.

[17] J. Feng, B. Xiao, R. Zhou, W. Pan, Acta Mater. 2013, 61, 7364.

[18] A. Meldrum, L. Boatner, R. Ewing, Phys. Rev. B 1997, 56, 13805.

[19] Y. Li, P. M. Kowalski, G. Beridze, A. Blanca-Romero, Y. Ji, V. L. Vinograd, J. D. Gale, D. Bosbach, Ceram. Trans. 2016, 255,165 .

[20] M. Robinson, N. A. Marks, K. R. Whittle, G. R. Lumpkin, Phys. Rev. B 2012, 85 .

[21] M. Robinson, N. A. Marks, G. R. Lumpkin, Mater. Chem. Phys. 2014, 147, 311.

[22] M. Robinson, N. A. Marks, G. R. Lumpkin, Phys. Rev. B 2012, 86.

[23] D. S. Aidhy, Y. Zhang, W. J. Weber, Scripta Mater. 2015, 98, 16.

[24] E. Gonzalez, Y. Abreu, C. M. Cruz, I. Pinera, A. Leyva, Nucl. Instrum. Meth. B 2015, 358, 142.

[25] B. Liu, H. Y. Xiao, Y. Zhang, D. S. Aidhy, W. J. Weber, J. PhysCondens. Matter 2013, 25.

[26] A. Merrill, C. D. Cress, J. E. Rossi, N. D. Cox, B. J. Landi, Phys. Rev. B 2015, 92. 
[27] W. Wu, S. Fahy, Phys. Rev. B 1994, 49, 3030.

[28] J. D. Gale, N. J. Henson, J. Chem. Soc., Faraday Trans. 1994, $90,3175$.

[29] S. Girard, J. D. Gale, C. Mellot-Draznieks, G. Ferey, Chem. Mater. 2001, 13, 1732.

[30] S. Plimpton, J. Comput. Phys. 1995, 117, 1.

[31] M. Bonomi, D. Branduardi, G. Bussi, C. Camilloni, D. Provasi, P. Raiteri, D. Donadio, F. Marinelli, F. Pietrucci, R. A. Broglia, M. Parrinello, Comput. Phys. Commun. 2009, 180, 1961 .

[32] J. Biersack, L. Haggmark, Nucl. Instrum. Methods 1980, 174, 257.

[33] J. F. Ziegler, M. Ziegler, J. Biersack, Nucl. Instrum. Meth. B 2010, 268, 1818, 19th International Conference on Ion Beam Analysis.

[34] M. T. Robinson, I. M. Torrens, Phys. Rev. B 1974, 9, 5008.

[35] m. T. Robinson, Radiat. Eff. Defect. S. 1994, 1, 3.

[36] S. Neumeier, Y. Arinicheva, N. Clavier, R. Podor, A. Bukaemskiy, G. Modolo, N. Dacheux, D. Bosbach, Prog. Nucl. Energ. 2016, .

[37] Y. Arinicheva, A. Bukaemskiy, S. Neumeier, G. Modolo, D. Bosbach, Prog. Nucl. Energ. 2014, 72, 144 .

[38] P. Kulriya, F. Singh, A. Tripathi, R. Ahuja, A. Kothari, R. Dutt, Y. Mishra, A. Kumar, D. Avasthi, Rev. Sci. Instrum. 2007, 78, 113901.

[39] P. Kulriya, R. Kumari, R. Kumar, V. Grover, R. Shukla A. Tyagi, D. Avasthi, Nucl. Instrum. Meth. B 2015, 342, 98.

[40] M. Lang, F. Zhang, R. Ewing, J. Lian, C. Trautmann, Z. Wang, Journal of Materials Research 2009, 24, 1322. 